\title{
Pro-inflammatory immune responses in leukocytes of premature infants exposed to maternal chorioamnionitis or funisitis
}

\author{
Courtney M. Jackson ${ }^{1}$, Casey B. Wells ${ }^{1}$, Meredith E. Tabangin'², Jareen Meinzen-Derr ${ }^{2}$, Alan H. Jobe ${ }^{3}$ and Claire A. Chougnet ${ }^{1}$
}

BACKGROUND: Acute chorioamnionitis contributes to premature birth, and is associated with postbirth complications. How chorioamnionitis impacts neonate's developing immune system has not been well defined.

METHODS: Blood from extremely preterm infants ( $\leq 28 \mathrm{wk}$ gestation) was drawn at the first, second, and fourth week of life. Blood was either left unstimulated or stimulated for $4 \mathrm{~h}$ with PMA/ionomycin. mRNA expression of transcription factors in unstimulated cells (RORC, TBET, GATA3, and Forkhead box protein 3 (FOXP3)) and inflammatory cytokines (IFN- $\boldsymbol{\gamma}$, TNF$\alpha, I L-2, I L-4, I L-5$, and IL-6) in unstimulated and stimulated cells were analyzed. Data were analyzed based on the diagnosis of chorioamnionitis, funisitis and bronchopulmonary dysplasia (BPD).

RESULTS: At 1 wk of life, exposure to funisitis, but not maternal chorioamnionitis was associated with an increased expression of RORC and RORC/FOXP3 ratio. These increases in RORC and RORC/FOXP3 ratio were sustained over the 4 wk of follow-up. Leukocytes from infants who developed BPD had increased stimulated and unstimulated IL-4 at the first week of life, but these increases were not sustained over time. In contrast, infants with mild BPD had a sustained decrease in stimulated IL-2.

CONCLUSION: Chorioamnionitis exposure, in particular to funisitis, lead to enhanced Th17-like responses that persist for 4 wk after birth. Infants who later developed BPD did not exhibit a strikingly distinct immune profile.

No eonatal $\mathrm{T}$ cell responses historically were thought to be deficient due to immaturity (1), although the supporting evidence came mainly from murine studies (2). However, more recent studies have demonstrated that human fetal development is a unique developmental stage in which fetal innate and adaptive responses are highly plastic and are influenced by in utero exposure to antigens or inflammatory mediators (3). Fetal T cells first appear in the human thymus at $\sim 10$ wk of gestational age (GA) (4), and the fetal $\mathrm{CD}^{+} \mathrm{T}$ cells are biased toward differentiation into regulatory $\mathrm{T}$ cells (5). However, a subset of $\mathrm{T}$ cells with a memory-like phenotype develops in the human fetus and displays inflammatory effector functions at birth (6). Other human fetal immune cells also appear to be functional. Notably, innate lymphoid cells (ILC) of different types have been identified in human fetuses (7). Fetal organs also contain KIR-expressing differentiated natural killer (NK) cells as early as $18 \mathrm{wk}$ GA (8). Mucosa-associated invariant $\mathrm{T}$ cells, which recognize microbial metabolites to produce proinflammatory cytokines such as interferon (IFN) $-\gamma$ and interleukin (IL)-17, have also been identified in the fetal small intestine, liver, and lung (9).

The development of the immune system of extremely low gestational age neonates (ELGANS) born before 28 wk of gestation is of particular interest. Many of these infants now survive, but they have increased prevalence of long-term inflammatory morbidities, notably bronchopulmonary dysplasia (BPD) and necrotizing enterocolitis (NEC) (10). In particular, BPD is a progressive lung injury syndrome with lung inflammation occurring prior to preterm birth and/or following birth and mechanical ventilation. Importantly, an estimated $25-40 \%$ of preterm births are associated with placental inflammation, which is often subclinical (11-13). This inflammation may be localized to the maternal placenta or membrane (chorioamnionitis) or progress to a fetal inflammatory response, as evidenced by cord inflammation (funisitis) and elevated cord plasma levels of IL-6 (14). This pattern has been reproduced experimentally in animal models; notably, fetal sheep and rhesus macaques respond to intra-amniotic LPS with skin, gut, and lung inflammation as well as systemic changes in immune cell phenotype (15-18). Although the fetus can be exposed to cytokines and microbial products through the amniotic fluid, the consequences of this in utero exposure to chorioamnionitis or funisitis on the developing infant immune system are poorly understood (18).

Most studies of human neonate immune responses, including those of preterm infants, are cross-sectional analyses using cord blood, due to blood sampling limitations in this

'Department of Pediatrics, Division of Immunobiology, Cincinnati Children's Hospital Research Foundation, University of Cincinnati College of Medicine, Cincinnati, Ohio; ${ }^{2}$ Division of Biostatistics and Epidemiology, Cincinnati Children's Hospital Medical Center, Cincinnati, Ohio; ${ }^{3}$ Department of Pediatrics Division of Neonatology/Pulmonary Biology, the Perinatal Institute, Cincinnati Children's Hospital Medical Center, University of Cincinnati, Cincinnati, Ohio. Correspondence: Claire Chougnet (Claire.Chougnet@cchmc.org) Received 17 May 2016; accepted 30 August 2016; advance online publication 14 December 2016. doi:10.1038/pr.2016.232 
population. Our objectives were to define the immune profile of infants at risk to develop BPD, and determine whether in-utero exposure to chorioamnionitis or funisitis influenced the neonate's immune responses. We thus chose a longitudinal approach using blood samples collected within the first, second, and fourth week of life. We measured the mRNA expression of a panel of proteins associated with immune responses. We included specific transcription factors associated with IL-17, IFN- $\gamma$, IL-4, and IL-5 producing immune cells (T cells, NK or ILC), namely RORC (the human ortholog of ROR $\gamma \mathrm{T}$ ), TBET, and GATA3. We also determined the expression of the transcription factor Forkhead box protein 3 (FOXP3), which is associated with regulatory $\mathrm{T}$ cells development and is a critical mediator of regulatory $\mathrm{T}$ cells suppressive activity. We also measured the induction of selected cytokine mRNAs (IFN$\gamma$, IL-2, IL-4, IL-5, TNF- $\alpha$, and IL-6) at baseline and after a short polyclonal stimulation. We used a quantitative reverse transcriptase (RT)-PCR approach on unseparated blood, since methods are now available to extract RNA for RT-PCR analysis from whole blood, without the need for separating mononuclear cells before stimulation $(19,20)$. Because quantitative RT-PCR is such a sensitive technique, a broad immune profile can be evaluated within the constraints of the extremely limited blood volumes that are available from preterm infants.

\section{METHODS}

\section{Patient Recruitment}

Mothers delivering $\leq 28 \mathrm{wk}$ gestation provided informed consent for collection of pregnancy related information and peripheral blood from their infants with a protocol approved by the IRB of Good Samaritan Hospital (Cincinnati, OH), University of Cincinnati Medical Center (Cincinnati, $\mathrm{OH}$ ) and Cincinnati Children's Hospital Medical Center (Cincinnati, OH). From 111 ELGANS, 35 were selected for this study because of availability of blood samples; the other 76 infants were not consented for blood. Mother and infant demographics were also collected.

\section{Diagnosis of BPD and Chorioamnionitis (Chorio)}

The infants were diagnosed with BPD as defined by the need for oxygen at $36 \mathrm{wk}$ GA (21). This group was then divided into infants who were on $<30 \%$ oxygen and low gas flows (mild BPD) and those on oxygen with gas flows $\geq 2$ liters/min or on oxygen and continuous positive airway pressure (severe BPD). No infant required mechanical ventilation at $36 \mathrm{wk}$.

The chorioamnion, placenta, and umbilical cord were scored for antenatal inflammation using a standardized procedure with histologic sections of chorioamnion, umbilical cord, and placenta stained with hematoxylin and eosin according to Redline's criteria (22). Fetal inflammation was defined by funisitis generating neutrophilic infiltration surrounding the umbilical cord vessels and Wharton's jelly. Three groups of infants were thus identified: no chorio, maternal chorio, and funisitis.

\section{Collection and Processing of Infant Blood}

Fresh blood from infants was collected in Microtainer tubes with dipotassium EDTA (BD Diagnostics, Sparks Glencoe, MD) during the first, second, and fourth wk of age, at the same time a clinical sample was needed. Within $4 \mathrm{~h}$ of collection, blood was diluted with an equal volume of complete medium (Roswell Park Memorial Institute (RPMI) 1640, supplemented with $10 \%$ heat-inactivated fetal calf-serum, HEPES, Glutamine) and divided into two equal aliquots: one left untreated and the other stimulated with PMA (50 ng/ $\mathrm{ml}$ ) (Sigma-Aldrich, St. Louis, MO) and ionomycin (1 $\mu \mathrm{g} / \mathrm{ml})$ (EMD Millipore, Billerica, MA). The cultures were incubated for $4 \mathrm{~h}$ at $37^{\circ} \mathrm{C}$. Red blood cells were lysed using $1 \mathrm{X}$ RBC Lysis Buffer according to the manufacturer's instructions (eBioscience, San Diego, CA) and the leukocytes were pelleted and stored at $-80^{\circ} \mathrm{C}$ until the extraction of mRNA.

\section{mRNA Amplification and Quantitative RT-PCR (qPCR) Analysis}

mRNA was isolated from unstimulated and stimulated leukocytes using the $\mu$ MACS mRNA Isolation kit (Miltenyi Biotec, Auburn, CA) and then amplified using the Ovation PicoSL WTA System V2 kit according to the manufacturer's instructions (NuGEN Technologies, San Carlos, CA). Amplified cDNA was purified with the MinElute Reaction Cleanup kit (Qiagen, Valencia, CA) then quality and quantity analyzed using a Bioanalyzer 2100 system (Qiagen; Agilent, Wilmington, DE). cDNA from unstimulated and stimulated samples was quantified for 12 target genes in eight separate panels by qPCR using TaqMan probes (TIB Molbiol LLC, Adelphia, NJ) and the LightCycler 480 Probes Master kit on the LightCycler 480 Instrument II (Roche Diagnostics, Indianapolis, IN). Transcription factor mRNA (RORC, TBET, GATA3, and FOXP3) were analyzed only in the unstimulated fraction, while the remaining target mRNA (CD3, IFN$\gamma$, IL-5, IL-2, IL-4, IL-6, and TNF- $\alpha$ ) were analyzed for both unstimulated and stimulated fractions to measure activation. Ubiquitin ligase (UBE2D2) mRNA expression was used for housekeeping. Primer and TaqMan probe information is given in Supplementary Table S1 online. TaqMan probes were used in either monoplex or duplex reactions; for duplex reactions, color compensation was performed using LightCycler 480 II software according to the manufacturer's instructions (Roche).

\section{Statistical Analysis}

Demographic data for the preterm infants are reported as percent of the population or mean \pm SEM. Differences in steroid treatment prevalence between groups were tested using Fisher's Exact test. Statistical analysis of qPCR data was performed using Prism 6.0 software for Windows (Graphpad, La Jolla, CA). For analyses of data for the first week of life, normality was checked for comparisons between groups. When the data were normally distributed, unpaired $t$-tests were used for comparisons of no chorio vs. any chorio and no BPD vs. all BPD, the Mann-Whitney $U$-test was used. When there were significant differences in the initial 2-group comparisons, BonferroniHolm adjustments were used to account for the multiple comparisons between no chorio, maternal chorio, and funisitis groups; and no BPD, mild BPD, severe BPD groups. To examine the repeated measures over time using linear models, Box-cox transformations were used to determine the optimal power transformation for each cytokine or transcription factor. GEE models were constructed on the transformed data (natural logarithm: RORC, FOXP3, IFN- $\gamma$, IL-5, and TNF- $\alpha$ or power: $(\text { TBET) })^{0.25}$, (GATA3) $)^{0.25}$, (IL-2) ${ }^{-0.25}$ ) to test for association between cytokine and transcription factor levels and the medical diagnoses of chorio, funisitis, and BPD. GEE models account for the within-subject correlation of repeated observations on individuals. Separate models were tested for funisitis, chorioamnionitis without funisitis, and BPD (mild vs. no BPD and severe BPD vs. mild $\mathrm{BPD}$ vs. no $\mathrm{BPD}$ ). We examined characteristics of missing data and tested for association with severity of chorioamnionitis, funisitis, and BPD. Multiple imputation procedures were used to examine potential effects of missing data. A data augmentation algorithm was used to generate 50 copies of the data set, each of which contains different estimates of the missing values. GEE models were estimated on the filled-in data. Parameter estimates and standard errors were pooled and compared with the analytic model results. SAS version 9.3 was used to conduct the analysis (SAS Institute, Cary, NC). Statistical significance was accepted at $P<0.05$ and statistical trends at $P<0.1$.

\section{RESULTS}

Demographic and Clinical Characteristics of the Preterm Infants About 35 infants who were part of the NHLBI funded Prematurity Respiratory Outcomes Program (23) were included in this study based on availability of blood samples at Good Samaritan Hospital, University of Cincinnati Medical Center, and Cincinnati Children's Hospital Medical Center 


\section{Articles | Jackson etal.}

(Cincinnati, OH). They were characterized according to the diagnosis of histological chorioamnionitis (maternal chorio), or chorio plus funisitis (any chorio) (Table 1). All infants diagnosed with funisitis were also exposed to maternal chorio (Figure 1a). Infants were also classified according to BPD diagnosis and its severity (Figure $\mathbf{1 b}$ ). Blood samples were available from 32 infants in the first week of life, 26 infants at the second week of life, and 14 infants in the fourth week of life. The majority of the infants were exposed to antenatal steroids prior to delivery, with no difference in treatment prevalence among the groups, $P>0.05$ (Table 1 ).

\section{Immune Profile for the First Week of Life}

All comparisons of chorio and funisitis groups were made to the no chorio group, and all comparisons of infants with BPD were made with the no BPD group. Additionally, the inflammatory markers were normalized to ubiquitin ligase (UBE2D2). As shown in Supplementary Figure S1a,b online, CD3 expression was similar in all the groups $(P>0.05)$. Infants exposed to any chorio had a trend toward higher expression of RORC mRNA (no chorio vs. any chorio, Mann-Whitney $U$-test, Bonferroni-Holm adjusted $P$-value $=0.05)$. Although FOXP3 mRNA expression was not significantly affected by chorio exposure (data not shown), the ratio of RORC to FOXP3 expression was higher in infants exposed to any chorio (no chorio vs. any chorio, adjusted $P=0.07$ ). Because chorio is a progressive condition that varies in severity, next we evaluated whether its severity influences neonatal immune responses. When RORC expression and RORC/FOXP3 ratio were analyzed comparing no chorio, maternal chorio, and funisitis infants respectively, only the subgroup of infants diagnosed with funisitis was different from the no chorio group whereas infants diagnosed with maternal chorio were not different (no chorio vs. funisitis, adjusted $P=0.08, P=0.05$; Figure 2a,b). Furthermore, exposure to any chorio resulted in a trend toward higher stimulated IFN- $\gamma$ mRNA expression (no chorio vs. any chorio, $P=0.09$ ). However, when we determined the contribution of maternal chorio and funisitis, the groups were not significantly different (Figure 2c). Similarly, infants with any chorio had a trend toward lower expression of IL-4 mRNA upon stimulation than the infants without any chorio $(P=$ 0.10 ); but analysis of funisitis vs. maternal chorio did not show evidence of significance (Figure 2d). No significant differences with maternal chorio or funisitis exposure in the first week of life were found for any of the other markers (GATA3, TBET, IL-2, IL-5, IL-6 or TNF- $\alpha$ mRNA with or without stimulation).

Table 1. Clinical characteristics of preterm infants selected for the study

\begin{tabular}{|c|c|c|c|c|c|c|c|c|c|}
\hline & \multirow[t]{2}{*}{ All infants } & \multicolumn{4}{|c|}{ Developed BPD } & \multicolumn{4}{|c|}{ Exposed to chorio } \\
\hline & & No BPD & All BPD & Mild BPD & $\begin{array}{c}\text { Severe } \\
\text { BPD }\end{array}$ & No chorio & $\begin{array}{c}\text { Any } \\
\text { chorio }\end{array}$ & $\begin{array}{l}\text { Maternal } \\
\text { chorio }\end{array}$ & Funisitis \\
\hline & $(n=35)$ & $(n=16)$ & $(n=19)$ & $(n=13)$ & $(n=6)$ & $(n=17)$ & $(n=18)$ & $(n=9)$ & $(n=9)$ \\
\hline Gestational age (GA) (Wk \pm SEM) & $26.5 \pm 0.2$ & $26.7 \pm 0.2$ & $26.2 \pm 0.3$ & $26.8 \pm 0.3$ & $25.1 \pm 0.4$ & $26.6 \pm 0.3$ & $26.4 \pm 0.3$ & $26.4 \pm 0.5$ & $26.32 \pm 0.4$ \\
\hline Birth Weight ( $\mathrm{g} \pm \mathrm{SEM})$ & $860 \pm 32$ & $903 \pm 43$ & $823 \pm 43$ & $873 \pm 56$ & $716 \pm 31$ & $888 \pm 48$ & $833 \pm 41$ & $865 \pm 65$ & $802 \pm 47$ \\
\hline Sex (\% Male) & 66 & 75 & 58 & 54 & 66 & 59 & 72 & 78 & 67 \\
\hline Race (\% Caucasian/African American/Asian) & $63 / 31 / 3$ & $63 / 31 / 6$ & $63 / 37 / 0$ & $85 / 15 / 0$ & $17 / 83 / 0$ & $76 / 24 / 0$ & $50 / 44 / 6$ & $56 / 33 / 11$ & $44 / 56 / 0$ \\
\hline Delivery mode (\% Vaginal) & 31 & 50 & 16 & 15 & 17 & 24 & 39 & 44 & 33 \\
\hline Use of antenatal steroids (\%) & 88 & 81 & 95 & 100 & 83 & 88 & 89 & 100 & 78 \\
\hline
\end{tabular}

a

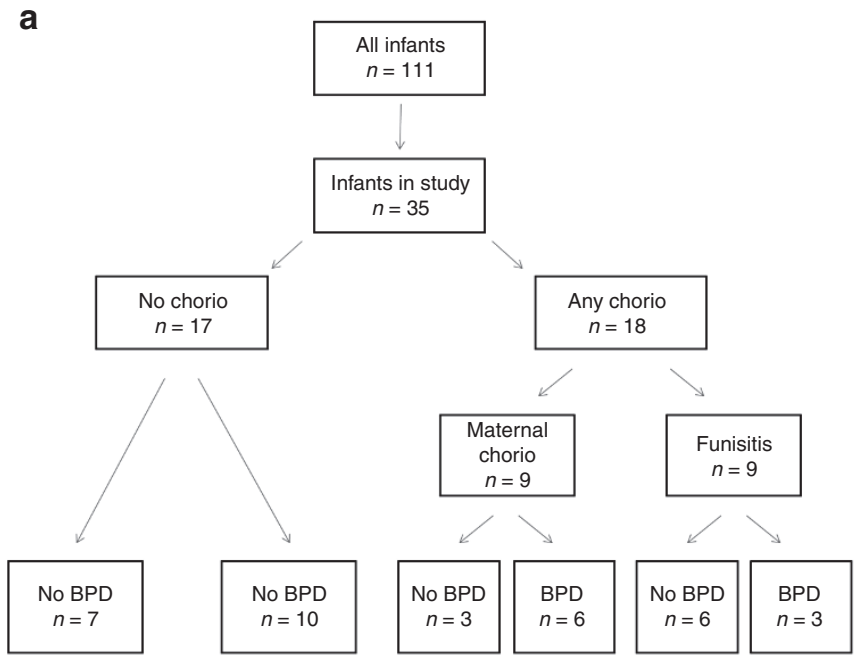

b

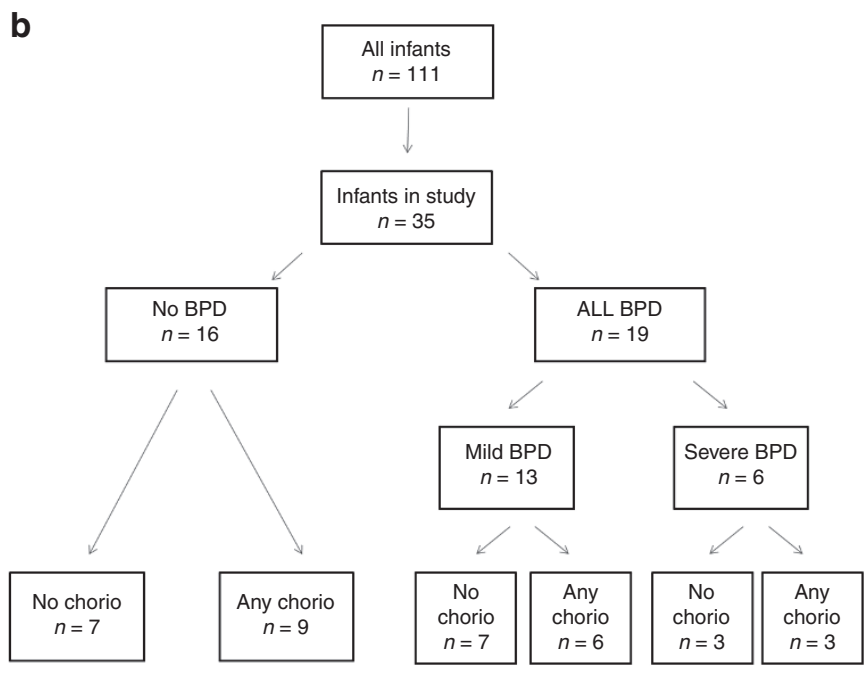

Figure 1. Tree diagrams depicting categorization of infants. Infants were partitioned by (a) chorio and funisitis diagnosis and (b) bronchopulmonary dysplasia (BPD) diagnosis. Mild BPD is oxygen use of $<30 \%$ at $36 \mathrm{wk}$; severe BPD is oxygen use $>30 \%$; and other ventilator support. 
In the first week of life, infants who were later diagnosed as having BPD and infants with mild BPD had a trend toward increased expression of IL- 4 mRNA in absence of stimulation compared with those who did not (no BPD vs. all BPD, adjusted $P=0.10$, no BPD vs. mild BPD adjusted $P=0.09$ ). However, there was no difference with severe BPD infants (Figure 3a). In addition, stimulated IL-4 mRNA expression was significantly increased in BPD infants (no BPD vs. all BPD, adjusted $P=$ 0.02 ), and this increase was particularly found in the severe
BPD group (adjusted $P=0.02$, Figure $3 \mathrm{~b}$ ). There was a trend toward an increased TBET/FOXP3 ratio with BPD (no BPD vs. all BPD adjusted $P=0.10$ ), but severe and mild BPD groups were similar (Figure 3c) [AU: Please note that panel $\mathbf{d}$ is not described in Figure 3, so panel $\mathbf{d}$ is changed as panel $\mathbf{c}$ is appropriate.] No other significant differences associated with BPD were found for any of the other markers (GATA3, FOXP3, IL-5, IL- 6 or TNF- $\alpha$ mRNA with or without stimulation).

\section{b}
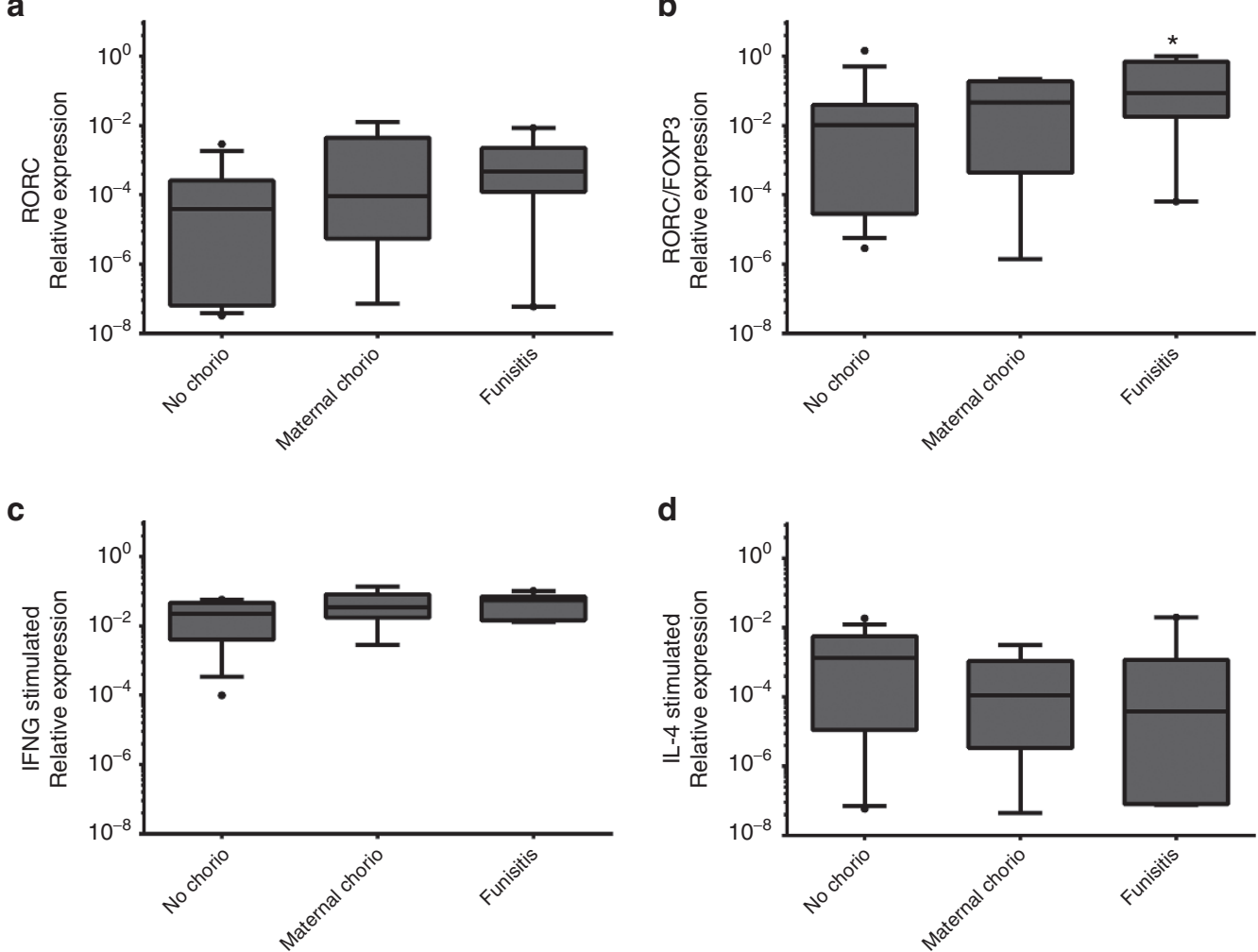

Figure 2. Gene expression levels of immune targets in preterm infants with or without chorio and/or funisitis in the first week of life. Relative gene expression of (a) RORC $P=0.08$ (no chorio vs. funisitis), (b) RORC/FOXP3 ratio $P=0.05$ (no chorio vs. funisitis), (c) IFNG stimulated and (d) IL4 stimulated in no chorio $(n=17)$, maternal chorio $(n=9)$ and funisitis $(n=9)$ groups. Groups were compared using unpaired $t$-tests when data were normally distributed; otherwise Mann-Whitney U-test was used. When there were significant differences in the initial 2-group comparisons (no chorio vs. any chorio), Bonferroni-Holm corrections were used to account for the multiple comparisons (no chorio vs. maternal chorio and no chorio vs. funisitis), ${ }^{*} P \leq 0.05$.

a

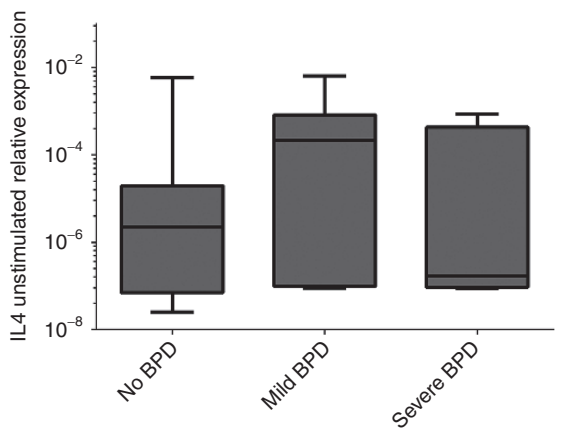

b

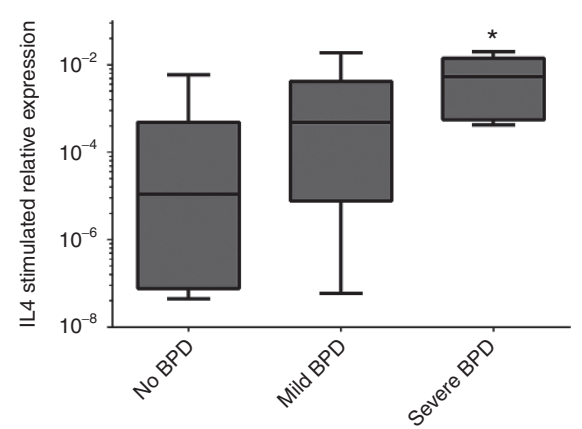

C

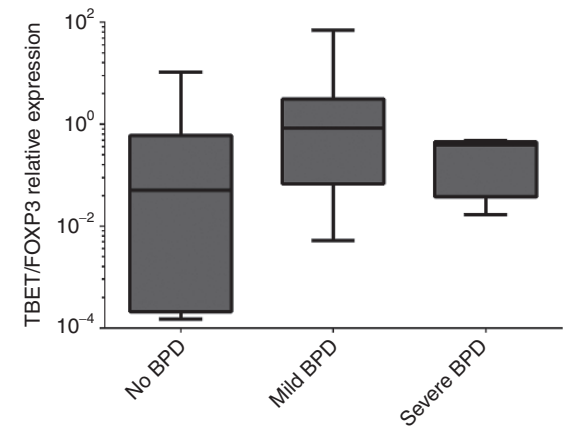

Figure 3. Gene expression levels of immune targets in preterm infants with or without bronchopulmonary dysplasia (BPD) in the first week of life. Relative gene expression of (a) IL-4 unstimulated, (b) IL-4 stimulated $P=0.02$ (no BPD vs. severe BPD), and (c) TBET/FOXP3 ratio in no BPD ( $n=16$ ), mild BPD $(n=13)$ and severe BPD $(n=6)$ groups. Groups were compared using unpaired $t$ - tests when data were normally distributed; otherwise MannWhitney $U$-test was used. When there were significant differences in the initial 2-group comparisons (no BPD vs. all BPD), Bonferroni-Holm corrections were used to account for the multiple comparisons (no BPD vs. mild BPD and no BPD vs. severe BPD), ${ }^{*} P \leq 0.05$. 


\section{Articles | Jackson et al.}

To eliminate the possibility that chorio may be a confounding factor in the analysis of BPD infants, we next excluded infants with chorio or funisitis from the analysis of BPD. Stimulated expression of IFN- $\gamma$ was increased in infants who were later diagnosed with BPD $(P=0.06$, Supplementary Figure S2a online). Higher expression of stimulated IL-4 mRNA and TBET/FOXP3 ratio were still found in this secondary analysis (Supplementary Figure S2b,c online).

\section{Change in Immune Profile Over Age of Infants}

To analyze differences between groups over the first 4 wk of life, repeated measures models were constructed to test for the association of cytokine and transcription factor expression with chorio, funisitis, and BPD. Regression coefficients (betas) estimated from generalized estimating equation (GEE) models were used to measure the average change in immune profile associated with the diagnosis. There was a robust and sustained increase in the expression of RORC mRNA and the RORC/FOXP3 ratio associated with funisitis over the 4-wk period (beta $=2.50, P=0.004$, Figure $4 \mathrm{a}$; and beta $=2.51, P$ $=0.001$, Figure $4 \mathrm{~b}$ respectively). Again, this increase was only seen with funisitis and not with maternal chorio (Figure 4a,b). FOXP3, TBET/FOXP3, TBET, GATA3, GATA3/FOXP3, IFN$\gamma$, TNF- $\alpha$, IL-2, IL-4, IL-6 or IL-5 were not significantly associated with funisitis or maternal chorio in this longitudinal analysis.

The development of BPD was characterized by a significant decrease in the expression of stimulated IL-2 mRNA in BPD infants compared with infants without BPD (beta for transformed IL- $2^{-0.25}=0.62, P=0.04$, Figure $4 d$ ). Compared with no BPD, slightly decreased levels of TBET was evident but not significantly associated with mild BPD (beta for transformed TBET $^{0.25}=-0.004, P=0.09$ ) or severe BPD (beta for transformed TBET $T^{0.25}=-0.01, P=0.73$, Figure $\left.4 \mathrm{c}\right)$. No significant
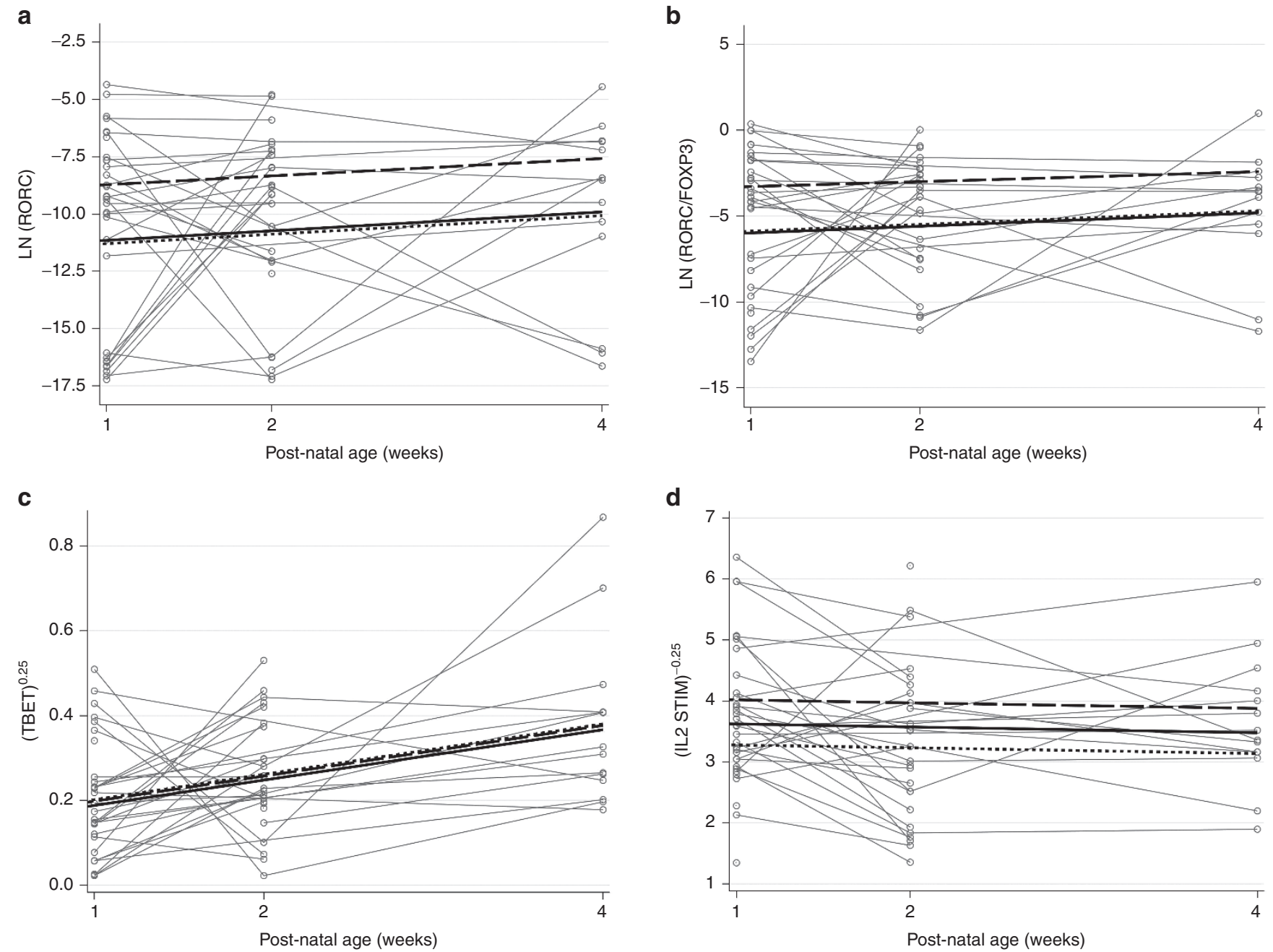

Figure 4. Changes in gene expression levels of immune targets over infant age for the first 4 wk of life in preterm infants with chorio, funisitis, or bronchopulmonary dysplasia (BPD) using Generalized Estimating Equations (GEE) models. Changes in relative gene expression were determined for (a) natural logarithm of RORC (beta $=2.50, P=0.04$ ) and (b) the natural logarithm of the RORC/FOXP3 ratio (beta $=2.51, P=0.001)$ in no chorio $(n=17$ ) (dotted line), maternal chorio $(n=9)$ (solid line), and funisitis $(n=9)$ (long dashed line) groups. Changes in relative gene expression were determined for (c) transformed TBET (mild BPD, beta for transformed (TBET) $)^{0.25}=-0.004, P=0.09$; severe BPD, beta for transformed $\left.(\text { TBET })^{0.25}=-0.01, P=0.73\right)$ and $(\mathbf{d}$ ) transformed stimulated IL-2 (beta for transformed IL-2-0.25 $=0.62, P=0.04$ in no BPD ( $n=16)$ (dotted line), mild BPD ( $n=13$ ) (long dashed line), and severe $\operatorname{BPD}(n=6)$ (solid line). 
associations were found for FOXP3, RORC, RORC/FOXP3, TBET/FOXP3, GATA3, GATA3/FOXP3, IFN- $\gamma$, TNF- $\alpha$, IL-2, IL-5, IL-4, or IL-6 in this longitudinal analysis.

\section{DISCUSSION}

We evaluated the fetal immune response to chorioamnionitis or funisitis, because fetal inflammation is very common and has been associated with brain, lung, and intestinal injury (24). Chorioamnionitis is defined as inflammation of the membranes shared between the mother and the developing fetus; whereas funisitis is inflammation within the umbilical cord. Previous studies that have examined immune responses of neonates to inflammation have focused on chorio and did not distinguish between funisitis and chorio (25).

In our study, funisitis, not maternal chorio, was associated with the increased expression of RORC mRNA, indicating that funisitis acts on the population of cord blood RORC ${ }^{+}$ cells that have been described in neonates born from normal pregnancies $(6,26)$. In the cord blood, a small subset of CCR $6^{+}$ or $\mathrm{CD}_{161} 1^{+}$effector memory $\mathrm{T}$ cells $\left(\mathrm{T}_{\mathrm{EM}}\right)$ has a profile of proTH17 cells, expressing RORC, IL-1R, and IL-23R $(6,26)$. These cells produce IL-17 but only if they are activated in the presence of IL-1 $\beta$ and IL-23 $(6,26)$. It is thus possible that the proinflammatory cytokine profile created by severe chorio as evidenced by elevated serum IL-1 $\beta$, IL- 6 , and IL- 8 levels (27), could activate or expand these pro-Th17 cells. Of note, our recent study of fetal inflammation in a rhesus macaque model supports this hypothesis, as we observed a transient increase in IL- $17^{+}$cells, mainly in the fetal spleen and lymph nodes (28).

In addition to increased RORC expression, we also found a trend toward increased stimulated IFN- $\gamma$ mRNA expression in infants exposed to any chorio. However, the reduced number of infants in each group likely did not provide enough power to definitively determine the contribution of maternal chorio or funisitis. However, this increase suggests the existence of a subset of cells that can develop a Th1 phenotype early in life as described by Zhang et al., and this response may be exacerbated in the presence of inflammation $(6,29,30)$.

Changes over time may identify potential biomarkers for BPD since BPD develops postnatally. An objective of our study was thus to determine whether a specific immune profile existed in infants that were developing BPD. Our results do not support such concept, as there were no striking differences in the immune profile of infants who developed BPD, including those who developed severe BPD vs. those who did not. This finding is in agreement with the fact that the $\mathrm{CD} 4^{+} \mathrm{T}$ cells from neonates who did or did not develop BPD were found to express similar levels of many cytokines, including IL-6 (25). In addition, there was a trend of increased TBET expression at the first week; but over time TBET appears to be similar among groups. Stimulated IL-4 appears to be increased in severe BPD infants. Although IL-4 may not play a major role in BPD pathogenesis, because low levels are found in the tracheal aspirates of BPD infants (31). Elevated levels may be associated with lung pathologies such as asthma, through IL-4 actions on lung fibroblast development (32). We did find that stimulated IL-2 mRNA levels were decreased in BPD infants over the $4 \mathrm{wk}$ of evaluation. This decrease in IL-2 corresponds to the increase in transformed stimulated (IL-2) - $^{-0.25}$ from the GEE model in neonates with mild BPD displayed in Figure 4d. The small sample number of samples with severe BPD may have limited the ability to detect this trend in this subgroup. This result is in concordance with reports of a decrease in circulating $\mathrm{CD} 4^{+}$ cells in BPD infants $(25,33)$, suggesting activation of the adaptive immune system and possible mobilization into the lung. On the other hand, there has been a report of a higher proportion of activated circulating $\mathrm{CD} 4^{+} \mathrm{T}$ cells observed in $\mathrm{BPD}$ infants (33). However, we did not find changes in CD3 mRNA expression with BPD (see Supplementary Figure S1b online).

While a strength of the study was the longitudinal analysis of neonatal samples, there were missing samples particularly at the 4 -wk time point. Available samples were similarly distributed across chorioamnionitis, funisitis, and BPD subgroups. GEE models incorporating the missing samples were similar to models using samples we had available

We chose to analyze neonatal blood, as compared with other studies that used cord blood, because it allowed us to examine the developing immune profile of neonates over the first week of life, something that had not yet been done. However, this strategy has limitations because we were only able to get a limited volume of blood, preventing us from determining the cellular sources of RORC and IFN- $\gamma$. Likewise, we were unable to measure IL-17 mRNA levels in our samples, as we found that whole blood profiling was too insensitive to detect IL-17 mRNA in both neonates and adult blood. Increased expression of RORC at the very least in our study denotes the possibility of an emerging TH17-like cell population since RORC is a crucial mediator of its differentiation (34). However, no conclusion about the absence or presence of IL-17 can be drawn strictly based off increased RORC mRNA. Another acknowledged limitation is the fact that the blood may not fully reflect what is happening in fetal organs. Indeed, we have shown in animal models of chorioamnionitis that the proinflammatory responses measured in the blood were blunted compared with those developing in lymphoid organs or lungs (18). This important caveat has to be kept in mind for all studies of neonatal responses using blood.

Overall, our findings demonstrate that ELGANS have the capacity to respond to in utero inflammation with a proinflammatory TH1/TH17 like phenotype. While these responses may be protective against pathogens, they may also contribute to the fetal inflammatory response syndrome, which involves multiorgan inflammation and injury (14). Moreover, our studies emphasize the important concept that funisitis and localized maternal chorioamnionitis do not have the same impact on the developing fetal immune system, and future studies of fetal inflammation need to clearly distinguish the two pathologies.

\section{SUPPLEMENTARY MATERIAL}

Supplementary material is linked to the online version of the paper at http:// www.nature.com/pr 


\section{Articles | Jackson et al.}

\section{STATEMENT OF FINANCIAL SUPPORT}

This work was supported by National Institutes of Health (Bethesda, MD) grant U01 HL101800 (to C.A.C. and A.H.J.) and Eunice Kennedy Shriver National Institute of Child Health and Human Development (Bethesda, MD) diversity supplement RO1HD078127-03 (to C.M.J.).

Disclosure: There is no conflict of interest to be reported.

\section{REFERENCES}

1. Maródi L. Innate cellular immune responses in newborns. Clin Immunol 2006;118:137-44

2. Adkins B, Leclerc C, Marshall-Clarke S. Neonatal adaptive immunity comes of age. Nat Rev Immunol 2004;4:553-64.

3. Mold JE, McCune JM. Immunological tolerance during fetal development: from mouse to man. Adv Immunol 2012;115:73-111.

4. Miller LH, Haynes JD, McAuliffe FM, Shiroishi T, Durocher JR, McGinniss $\mathrm{MH}$. Evidence for differences in erythrocyte surface receptors for the malarial parasites, Plasmodium falciparum and Plasmodium knowlesi. J Exp Med 1977;146:277-81.

5. Mold JE, Venkatasubrahmanyam S, Burt TD, et al. Fetal and adult hematopoietic stem cells give rise to distinct $\mathrm{T}$ cell lineages in humans. Science 2010;330:1695-9.

6. Zhang X, Mozeleski B, Lemoine S, et al. CD4 T cells with effector memory phenotype and function develop in the sterile environment of the fetus. Sci Transl Med 2014;6:238ra72.

7. Hazenberg MD, Spits H. Human innate lymphoid cells. Blood 2014;124:700-9.

8. Ivarsson MA, Loh L, Marquardt N, et al. Differentiation and functional regulation of human fetal NK cells. J Clin Invest 2013;123:3889-901.

9. Leeansyah E, Loh L, Nixon DF, Sandberg JK. Acquisition of innate-like microbial reactivity in mucosal tissues during human fetal MAIT-cell development. Nat Commun 2014;5:3143.

10. Eichenwald EC, Stark AR. Management and outcomes of very low birth weight. N Engl J Med 2008;358:1700-11.

11. Goldenberg RL, Culhane JF, Iams JD, Romero R. Epidemiology and causes of preterm birth. Lancet 2008;371:75-84.

12. Romero R, Gotsch F, Pineles B, Kusanovic JP. Inflammation in pregnancy: its roles in reproductive physiology, obstetrical complications, and fetal injury. Nutr Rev 2007;65(12 Pt 2):S194-202.

13. Stoll BJ, Hansen NI, Higgins RD, et al.; National Institute of Child Health and Human Development. Very low birth weight preterm infants with early onset neonatal sepsis: the predominance of gram-negative infections continues in the National Institute of Child Health and Human Development Neonatal Research Network, 2002-2003. Pediatr Infect Dis J 2005;24:635-9.

14. Gomez R, Romero R, Ghezzi F, Yoon BH, Mazor M, Berry SM. The fetal inflammatory response syndrome. Am J Obstet Gynecol 1998;179:194-202.

15. Kallapur SG, Nitsos I, Moss TJ, et al. IL-1 mediates pulmonary and systemic inflammatory responses to chorioamnionitis induced by lipopolysaccharide. Am J Respir Crit Care Med 2009;179:955-61.

16. Wolfs TG, Buurman WA, Zoer B, et al. Endotoxin induced chorioamnionitis prevents intestinal development during gestation in fetal sheep. PLoS One 2009;4:e5837.

17. Kemp MW, Saito M, Nitsos I, Jobe AH, Kallapur SG, Newnham JP. Exposure to in utero lipopolysaccharide induces inflammation in the fetal ovine skin. Reprod Sci 2011;18:88-98.
18. Kallapur SG, Presicce P, Rueda CM, Jobe AH, Chougnet CA. Fetal immune response to chorioamnionitis. Semin Reprod Med 2014;32:56-67.

19. Daegelmann C, Herberth G, Röder S, et al.; LISAplus study group. Association between suppressors of cytokine signalling, T-helper type 1/T-helper type 2 balance and allergic sensitization in children. Clin Exp Allergy 2008;38:438-48.

20. Herberth G, Daegelmann C, Weber A, et al.; LISAplus Study Group. Association of neuropeptides with Th1/Th2 balance and allergic sensitization in children. Clin Exp Allergy 2006;36:1408-16.

21. Shennan AT, Dunn MS, Ohlsson A, Lennox K, Hoskins EM. Abnormal pulmonary outcomes in premature infants: prediction from oxygen requirement in the neonatal period. Pediatrics 1988;82:527-32.

22. Redline RW, Faye-Petersen O, Heller D, Qureshi F, Savell V, Vogler C; Society for Pediatric Pathology, Perinatal Section, Amniotic Fluid Infection Nosology Committee. Amniotic infection syndrome: nosology and reproducibility of placental reaction patterns. Pediatr Dev Pathol 2003;6:435-48.

23. Pryhuber GS, Maitre NL, Ballard RA, et al.; Prematurity and Respiratory Outcomes Program Investigators. Prematurity and respiratory outcomes program (PROP): study protocol of a prospective multicenter study of respiratory outcomes of preterm infants in the United States. BMC Pediatr 2015;15:37.

24. Gantert M, Been JV, Gavilanes AW, Garnier Y, Zimmermann LJ, Kramer BW. Chorioamnionitis: a multiorgan disease of the fetus? J Perinatol 2010;30 Suppl:S21-30.

25. Misra RS, Shah S, Fowell DJ, et al. Preterm cord blood CD $4 \rrbracket T$ cells exhibit increased IL-6 production in chorioamnionitis and decreased CD4区 T cells in bronchopulmonary dysplasia. Hum Immunol 2015;76:329-38.

26. Cosmi L, De Palma R, Santarlasci V, et al. Human interleukin 17-producing cells originate from a CD161+CD4+ T cell precursor. J Exp Med 2008;205:1903-16

27. Nishimaki S, Shima Y, Sato M, An H, Kadota K, Yokota S. Postnatal changes of cytokines in premature infants with or without funisitis. J Matern Fetal Neonatal Med 2014;27:1545-9.

28. Kallapur SG, Presicce P, Senthamaraikannan P, et al. Intra-amniotic IL-1 $\beta$ induces fetal inflammation in rhesus monkeys and alters the regulatory $\mathrm{T}$ cell/IL-17 balance. J Immunol 2013;191:1102-9.

29. Matsuoka T, Matsubara T, Katayama K, Takeda K, Koga M, Furukawa S. Increase of cord blood cytokine-producing $\mathrm{T}$ cells in intrauterine infection. Pediatr Int 2001;43:453-7.

30. Romero R, Chaemsaithong P, Korzeniewski SJ, et al. Clinical chorioamnionitis at term II: the intra-amniotic inflammatory response. J Perinat Med 2016;44:5-22.

31. Baier RJ, Loggins J, Kruger TE. Interleukin-4 and 13 concentrations in infants at risk to develop Bronchopulmonary Dysplasia. BMC Pediatr 2003;3:8.

32. Sempowski GD, Beckmann MP, Derdak S, Phipps RP. Subsets of murine lung fibroblasts express membrane-bound and soluble IL-4 receptors. Role of IL-4 in enhancing fibroblast proliferation and collagen synthesis. J Immunol 1994;152:3606-14.

33. Turunen R, Vaarala O, Nupponen I, et al. Activation of T cells in preterm infants with respiratory distress syndrome. Neonatology 2009;96:248-58.

34. Ivanov II, McKenzie BS, Zhou L, et al. The orphan nuclear receptor RORgammat directs the differentiation program of proinflammatory IL-17+ T helper cells. Cell 2006;126:1121-33. 